\title{
Voltammetric determination of zolpidem by using glassy carbon electrode modified with $\mathrm{Ag} / \mathrm{ZnO}$ nanoplates
}

\author{
Mohammad Reza Aflatoonian'a,b,*, Behnaz Aflatoonianc, Reza Alizadehd, \\ Reza Abbasi Rayenie \\ ${ }^{a}$ Research Center for Tropical and Infectious Diseases, Kerman University of Medical Sciences, \\ Kerman, Iran \\ ${ }^{b}$ Leishmaniasis Research Center, Kerman University of Medical Sciences, Kerman, Iran \\ cNeuroscience Research Center, Kerman University of Medical Sciences, Kerman, Iran \\ dDepartment of Chemistry, Faculty of Science, Qom University, Qom, Iran \\ eKerman University of Medical Sciences, Kerman, Iran
}

Received: 13 October 2019, Accepted: 02 November 2019, Published: 23 November 2019

\begin{abstract}
Zolpidem is an imidazopyridine derivative, non-benzodiazepine and sedative-hypnotic medicine. It is a widely prescribed medication in adults used for the short-term treatment of a sleep problem called insomnia and frequent awakenings. In this work, the zolpidem was determined using $\mathrm{Ag} / \mathrm{ZnO}$ nanoplates modified glassy carbon electrode (Ag-ZnO/GCE). Electrochemical behavior of the zolpidem was investigated by differential pulse voltammetry (DPV), chronoamperometry (CHA), and cyclic voltammetry $(\mathrm{CV})$ using the $\mathrm{Ag}-\mathrm{ZnO} / \mathrm{GCE}$. The results revealed that, the current responses of the zolpidem improved significantly due to the high catalytic activity and electron transfer reaction of nanoplates. The linear range of zolpidem was found to be from $0.1 \mu \mathrm{M}$ to $500.0 \mu \mathrm{M}$ with the detection limit of $0.03 \mu \mathrm{M}$. In addition, this original sensor showed numerous benefits such as reproducibility, high stability, and rapid response $(20 \mathrm{~s})$.
\end{abstract}

Keywords: Zolpidem; voltammetric determination; glassy carbon electrode; $\mathrm{Ag} / \mathrm{ZnO}$ nanoplates.

\section{Introduction}

Zolpidem is an imidazopyridine derivative and non-benzodiazepine sedative-hypnotic drug [1]. Zolpidem is structurally different from the benzodiazepines; however, they both have the same pharmacological effect. Zolpidem has a rapid onset of action. It has short half-life and anticonvulsant effects, and used for treatment of anxiety and sleep disorders [2]. Zolpidem has a highly selective interaction with omega1 receptor subtype belonging to the $\gamma$ aminobutyric acid (GABA)ergic system, which is mostly associated with sleep [35]. Zolpidem has been quantified in various biological samples using common analytical techniques including,

*Corresponding author: M.R. Aflatoonian

Tel: +98 3426226613 , Fax: +98 3426226617

E-mail: m.aflatoonian97@gmail.com

Eurasian Chem. Commun., (2020) 35-43 
high performance liquid chromatography [6], GC/MS [7], chemiluminescence [8], spectrophotometry

[9], radioimmunoassay chromatography $[10]$, [11], gas electrochemical methods $[12,13]$.

Majority of the approaches are time consuming and have entail inadequate sensitivity, made them complicated experiment procedures. Compared to other approaches, the electrochemical approaches exhibited some advantages such as enhanced sensitivity and selectivity. Thus, there is substantial focus on developing a straightforward, expeditious, cost-efficient, and sensitive electrochemical approach to detect analytes [14-25]. Due to the exceptional characteristics of the nanoparticles, they have been used in various industries. Several factors may impact nanoparticles structure including the adopted approach and temperature [26-42].

New platforms should meet some factors such as low detection limits, good stability, simplicity, low cost, and high speed. Moreover, prompt response is vital in most situations such as illness diagnostics which will entail faster treatment. Some of the techniques should be integrated under such new lenses. Electrochemical detection was found to be suitable to be combined with the paper based analytical device, as such combination may enhance analyte quantification, resulting in increased sensitivity and reduced detection limits as well as characteristics such as low costs, simplicity, portability, and miniaturization $[47,48]$.

To the best of our knowledge, no study has been reported so far on the determination of zolpidem by using Ag$\mathrm{ZnO}$ /GCE. In this research study, we report the preparation and application of $\mathrm{Ag}-\mathrm{ZnO} / \mathrm{GCE}$ for the determination of zolpidem without any additional modification such as addition of electron transfer mediator or specific reagents for the first time. The main objectives of this $\mathrm{Ag}-\mathrm{ZnO} / \mathrm{GCE}$ for determination of zolpidem are: wide linear dynamic range, short time of the procedure and no use of electron transfer mediator.

\section{Experimental}

Chemicals and apparatus

An Autolab potentiostat/galvanostat (PGSTAT 302N, Eco Chemie, the Netherlands) was utilized to conduct electrochemical measurements. The General Purpose Electrochemical System (GPES) software was used to control the defined experimental settings. A glassy carbon electrode (Azar Electrode, Urmia, Iran with $2 \mathrm{~mm}$ diameter) modified with $\mathrm{Ag} / \mathrm{ZnO}$ nanoplates as working electrode, an $\mathrm{Ag} / \mathrm{AgCl} / \mathrm{KCl} \quad\left(\begin{array}{ll}3.0 & \mathrm{M}) \text { reference }\end{array}\right.$ electrode (Azar Electrode, Urmia, Iran) and a platinum wire counter electrode (Azar Electrode, Urmia, Iran) are the parts that form the electrochemical cell. To measure the $\mathrm{pH}$, a $710 \mathrm{pH}$ meter metrohm was used.

Analytical grade zolpidem was used along with all other analytical grade reagents which were obtained from Merck, Darmstadt, Germany. Orthophosphoric acid was used to prepare buffer solution. The relevant salts were above 2.0-9.0 pH range. $\mathrm{Ag} / \mathrm{ZnO}$ nanoplates were synthesized as the procedure reported previously [49].

\section{Formation of electrode}

$\mathrm{Ag}-\mathrm{ZnO}$ nanoplates was applied as coating on the bare glassy carbon electrode. Dispersion of $1 \mathrm{mg}$ of $\mathrm{Ag}-\mathrm{ZnO}$ nanoplates with ultrasonication for $1 \mathrm{~h}$ was used to prepare a stock solution of $\mathrm{Ag}-\mathrm{ZnO}$ nanoplates in $1 \mathrm{~mL}$ of the aqueous solution, whereas $5 \mu \mathrm{L}$ of aliquots of the $\mathrm{Ag}-\mathrm{ZnO}$ nanoplates $/ \mathrm{H}_{2} \mathrm{O}$ suspension solution was cast on the glassy carbon electrode. Then, it 
remained up to the time of evaporation of the solvent in room temperature.

\section{Real specimen preparation}

Five tablets of zolpidem were grinding. By dissolving $100 \mathrm{mg}$ of the powder in $25 \mathrm{~mL}$ water using ultrasonication, the solution of tablet was prepared. Various amounts of this solution was transferred into cell and diluted with buffer solution. The amount of the drug in tablet was obtained using the standard addition method. Upon collecting urine samples, they were promptly kept in a refrigerator for $15 \mathrm{~min}$ at $2000 \mathrm{rpm}$ centrifugation was implemented for $10 \mathrm{~mL}$ of the samples. A $0.45 \mu \mathrm{m}$ filter was used to filter the supernatant. Then, various solution volumes were put into a $25 \mathrm{~mL}$ volumetric flask prior to being diluted with PBS of pH 7.0 to the mark. Various volumes of zolpidem were used to spike the diluted urine samples. The proposed method was used to analyse the zolpidem contents using the standard addition method.

\section{Results and discussion \\ Zolpidem electrochemical profile on Ag- $\mathrm{ZnO} / G C E$}

The electrochemical behaviour of zolpidem is dependent on the $\mathrm{pH}$ value of the aqueous solution (Figure 1). Thus, optimizing the solution $\mathrm{pH}$ would apparently be crucial for achieving zolpidem electro-catalytic oxidation. Therefore, $\mathrm{CV}$ was used to explore the zolpidem electrochemical behaviour in $0.1 \mathrm{M}$ PBS in diverse $\mathrm{pH}$-values $(2.0<\mathrm{pH}$ <9.0) on Ag-ZnO/GCE surface (Figure 2). Outputs revealed that, the zolpidem electro-catalytic oxidation at the Ag$\mathrm{ZnO} / \mathrm{GCE}$ surface has been very desirable under the neutral conditions compared to in the basic or acidic media. Therefore, such a condition would appear as the progressive development in the anodic peak current in the CVs equal to zolpidem. Finally, $\mathrm{pH}$ of 7.0 was targeted as an optimized $\mathrm{pH}$ for electrocatalyzing zolpidem oxidation at $\mathrm{Ag}-\mathrm{ZnO} / \mathrm{GCE}$ surface.<smiles>Cc1ccc(-c2nc3ccc(C)cn3c2CC(=O)N(C)C)cc1</smiles><smiles></smiles>

Figure 1. Electrochemical mechanism for oxidation of zolpidem 


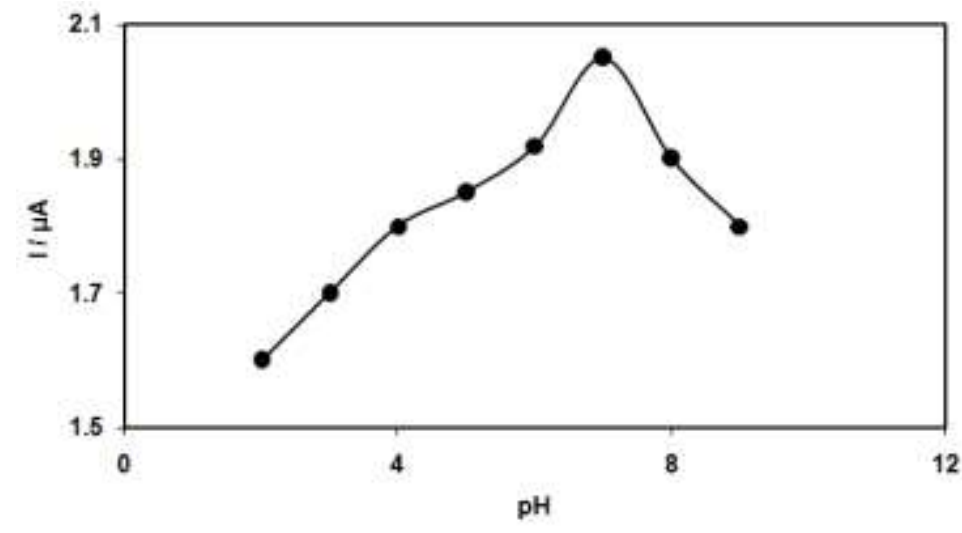

Figure 2. Plot of $I_{p}$ vs. pHs of buffer solution (2.0, 3.0, 4.0, 5.0, 6.0, 7.0, 8.0 and 9.0) in the presence of $200.0 \mu \mathrm{M}$ zolpidem

Figure 3 depicts the $100.0 \mu \mathrm{M}$ of zolpidem cyclic voltammograms acquired by applying the unadjusted GCE (Curve a) and Ag-ZnO/GCE (Curve b). Regarding the zolpidem oxidation at the adjusted electrode surface, the peak potential was at 900 $\mathrm{mV}$. This value is $200 \mathrm{mV}$ more negative compared to that of the unadjusted GCEs. This showed a significant improvement of the electrode performance toward zolpidem oxidation by changing the constant GCE with $\mathrm{Ag} / \mathrm{Zno}$ nanoplates. Curve c shows the $\mathrm{Ag}-\mathrm{ZnO} / \mathrm{GCE}$ in $0.1 \mathrm{M}$ PBS (pH 7.0) in the absence of zolpidem.

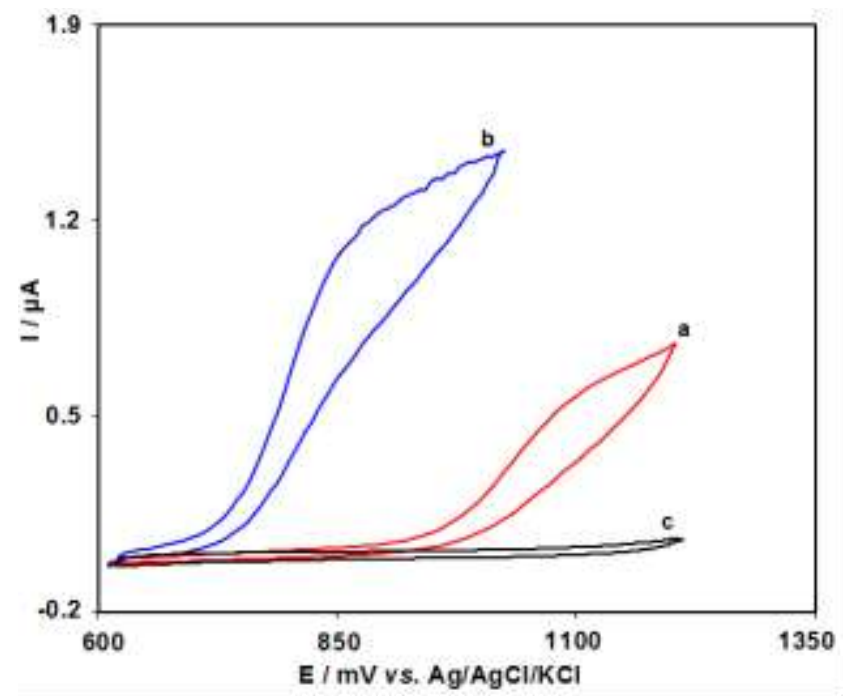

Figure 3. Cyclic voltammograms of (a) bare GCE and (b) Ag-ZnO/GCE in 0.1 M PBS (pH 7.0) in the presence of $100.0 \mu \mathrm{M}$ zolpidem at the scan rate $50 \mathrm{mVs}^{-1}$. Also curve c shows Ag-ZnO/GCE in $0.1 \mathrm{M}$ PBS (pH 7.0) in the absence of zolpidem

\section{Effect of scan rate}

Figure 4 depicts the impacts of possible scan rates on zolpidem oxidation currents, showing that by enhancing the scan rate, the peak currents enhanced.
Moreover, due to the linear Ip plot against the potential scan rate square root $\left(v^{1 / 2}\right)$ for the zolpidem, it was proven that oxidation procedure was diffusion regulated [50]. 


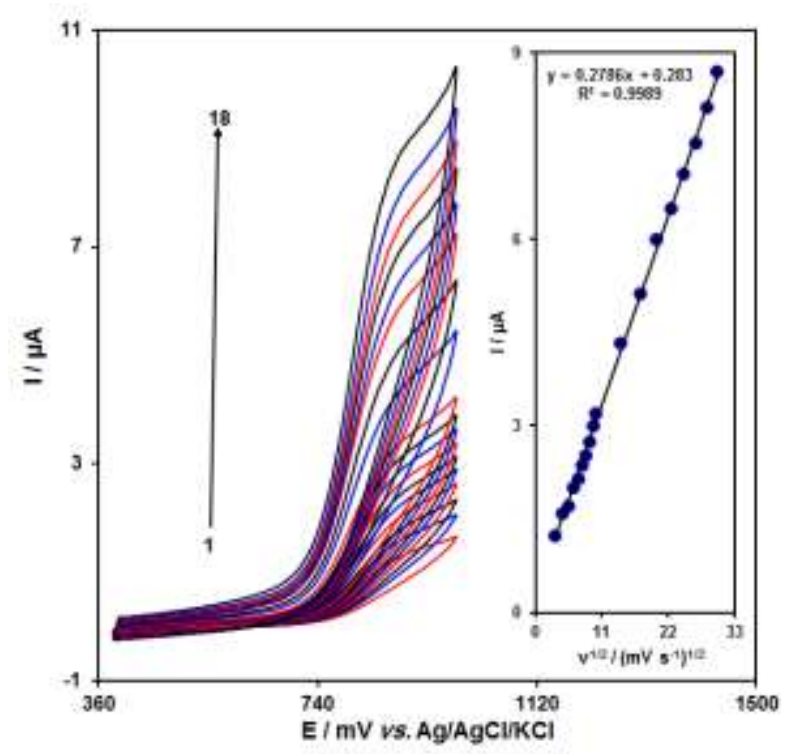

Figure 4. Cyclic voltammograms of Ag-ZnO/GCE in $0.1 \mathrm{M}$ PBS (pH 7.0) containing $200.0 \mu \mathrm{M}$ zolpidem at various scan rates; numbers 1-18 correspond to 10, 20, 30, 40, 50, 60, 70, 80, 90, 100, 200, $300,400,500,600,700,800$ and $900 \mathrm{mV} \mathrm{s}^{-1}$, respectively. Inset: variation of anodic peak current vs. $v^{1 / 2}$.

\section{Chronoamperometric analysis}

The zolpidem specimen' chronoamperometric analysis via the $\mathrm{Ag}$ $\mathrm{ZnO} / \mathrm{GCE}$ was conducted at $950 \mathrm{mV}$ and the outcomes for various zolpidem specimens in $\mathrm{PBS}, \mathrm{pH}=7.0$ are depicted in Figure 5. For electroactive materials' chronoamperometric analysis at transfer restricted circumstances, the Cottrell equation is as following (Equation 1).

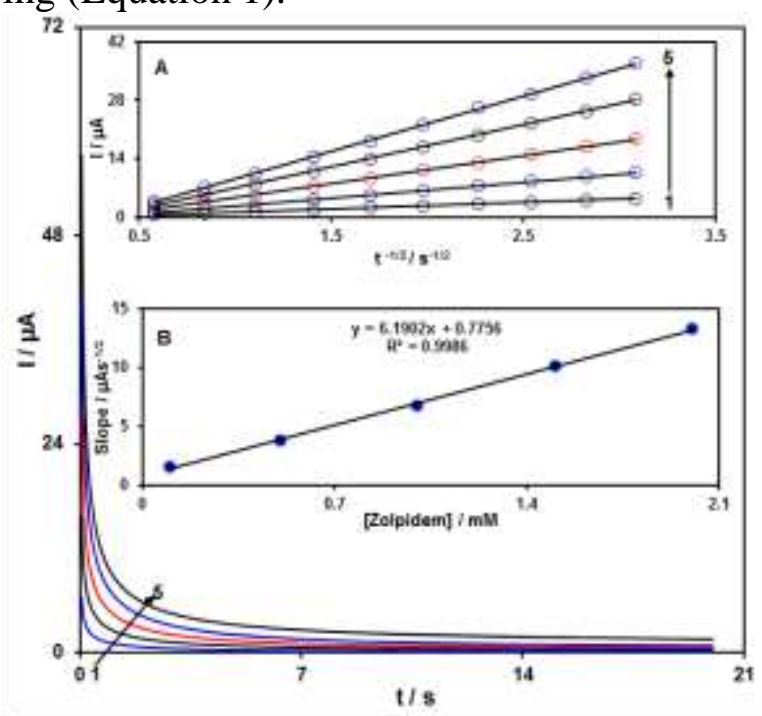

Figure 5. Chronoamperograms obtained at Ag-ZnO/GCE in 0.1 M PBS (pH 7.0) for different concentration of zolpidem. The numbers $1-5$ correspond to $0.1,0.5,1.0,1.5$ and $2.0 \mathrm{mM}$ of zolpidem. Insets: (A) Plots of I vs. $\mathrm{t}^{-1 / 2}$ obtained from chronoamperograms 1-5. (B) Plot of the slope of the straight lines against zolpidem concentration. 


\section{Calibration curve}

The zolpidem peak currents via the Ag$\mathrm{ZnO} / \mathrm{GCE}$ were applied to quantitatively analyse the zolpidem within water solutions. Due to the differential pulse voltammetry advantages concerning enhanced sensitivity and improved investigative utilization properties. The adjusted electrode was applied as a working electrode in DPV analysis within the zolpidem range solution in 0.1
M PBS (Step potential $=0.01 \mathrm{~V}$ and pulse amplitude $=0.025 \mathrm{~V}$ ). In regard to DPV of zolpidem via $\mathrm{Ag}-\mathrm{ZnO} / \mathrm{GCE}$, linear activity was evident within the $0.1-500.0$ $\mu \mathrm{M}$ range and correlation coefficient of 0.9994 (Figure 6). The relevant detection limit was $0.03 \mu \mathrm{M}$. Table 1 depicts a comparison of analytical properties for the detection of zolpidem at the prepared electrode in this work.

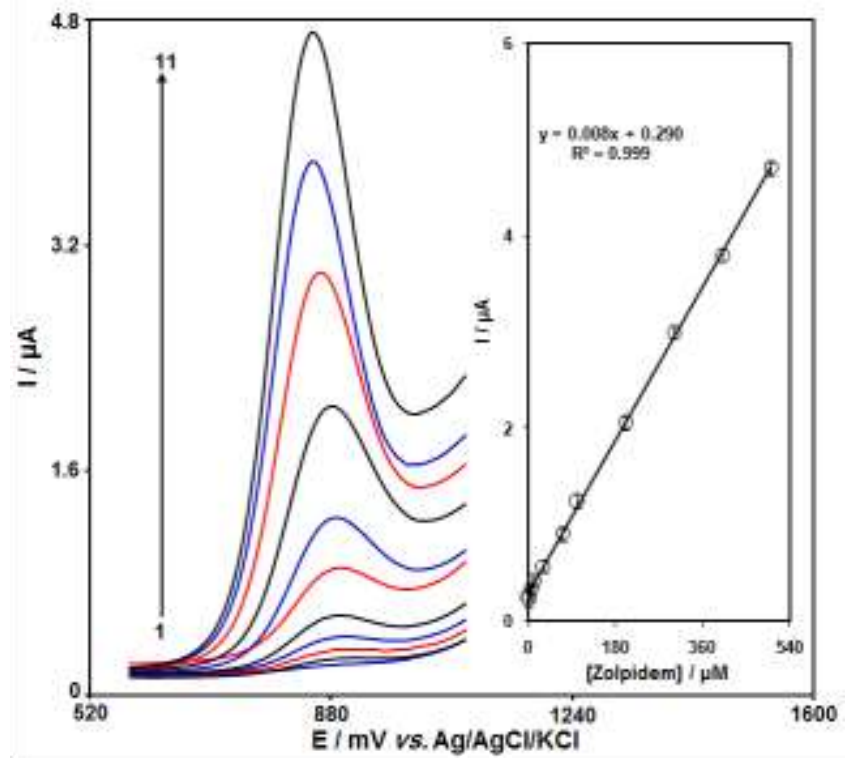

Figure 6. DPVs of Ag-ZnO/GCE in $0.1 \mathrm{M}(\mathrm{pH} 7.0)$ containing different concentrations of zolpidem. Numbers 1-11 correspond to $0.1,1.0,5.0,10.0,30.0,70.0,100.0,200.0,300.0,400.0$ and $500.0 \mu \mathrm{M}$ of zolpidem. Inset: plot of the peak current as a function of zolpidem concentration in the range of 0.1 $500.0 \mu \mathrm{M}$.

Table 1. A comparison of the efficiency of various modified electrodes reported for the detection of

\begin{tabular}{|c|c|c|c|c|}
\hline Electrode & Modifier & LOD $(\mu \mathrm{M})$ & $\operatorname{LDR}(\boldsymbol{\mu M})$ & Ref. \\
\hline $\begin{array}{l}\text { Pencil Graphite } \\
\text { Electrode }\end{array}$ & - & 1.0 & $10-30$ & 12 \\
\hline $\begin{array}{l}\text { Glassy Carbon } \\
\text { Electrode }\end{array}$ & - & 0.2 & $0.5-10$ & 13 \\
\hline $\begin{array}{l}\text { Glassy Carbon } \\
\text { Electrode }\end{array}$ & $\mathrm{Ag} / \mathrm{ZnO}$ nanoplates & 0.03 & $0.1-500.0$ & This work \\
\hline
\end{tabular}

\section{Interference studies}

This study examined the effects of diverse materials as the compounds with a potential interference with zolpidem detection under the optimized conditions with $50.0 \mu \mathrm{M}$ zolpidem at $\mathrm{pH}=7.0$. It should be noted that, the potentially interfering materials have been selected from a group of materials that are usually observed with zolpidem in the biological fluids. Moreover, the limit of tolerance was described as the highest concentration of the interfering material with the standard deviation of $\pm 5 \%$. As shown by the outputs, glucose, NADH, acetaminophen, uric acid, dopamine, epinephrine, norepinephrine, isoproterenol, lactose, saccarose, 
fructose, benzoic acid, methanol, ethanol, urea, caffeine, $\mathrm{Ca}^{2+}, \mathrm{Mg}^{2+}, \mathrm{Al}^{3+}$, $\mathrm{NH}_{4}{ }^{+}, \mathrm{Fe}^{+2}, \mathrm{Fe}^{+3}, \mathrm{~F}^{-}, \mathrm{SO}_{4}{ }^{2-}$ and $\mathrm{S}^{2-}$ had no interference with the zolpidem detection.

Repeatability and stability of $\mathrm{Ag}$ $\mathrm{ZnO} / G C E$

Long-term stability of the Ag-ZnO/GCE was assessed over a 3-week period. The CVs were recorded after the modified electrode was stored in atmosphere at room temperature. The peak potential for zolpidem oxidation was found to be unchanged and the current signals revealed less than $2.3 \%$ decrease relative to the initial response. The antifouling properties of the modified electrode toward zolpidem oxidation and its oxidation products were investigated by recording the CVs of the modified electrode prior and after being used at the presence of the zolpidem. The CVs were recorded at the presence of the zolpidem after having cycled the potential 15 times at a scan rate of $50 \mathrm{mV} \cdot \mathrm{s}^{-1}$. The peak potentials were unchanged and the currents decreased by less than $2.2 \%$. Therefore, the sensitivity of the Ag$\mathrm{ZnO} / \mathrm{GCE}$ increased, and the fouling effect of the analyte and its oxidation decreased.

\section{Analysis of real samples}

To evaluate the zolpidem within the real specimens, the presented approach was implemented to determine zolpidem in the form of tablet sample and urine. Thus, the standard addition method was implemented for this analysis and the outcomes are presented in Table 2. The acquired zolpidem recoveries were found to be adequate and the reproducibility of the results was on the basis of the mean relative standard deviation (RSD).

Table 2. The application of $\mathrm{Ag}-\mathrm{ZnO} / \mathrm{GCE}$ for determination of zolpidem in real samples $(\mathrm{n}=5)$. All

\begin{tabular}{ccccc}
\multicolumn{5}{c}{ concentrations are in $\mu \mathrm{M}$. } \\
\hline Sample & Spiked & Found & Recovery (\%) & R.S.D. (\%) \\
\hline \multirow{4}{*}{ Tablet } & 0 & 5.0 & - & 3.2 \\
& 2.5 & 7.6 & 101.3 & 2.2 \\
& 5.0 & 9.7 & 97.0 & 2.4 \\
& 7.5 & 12.4 & 99.2 & 1.7 \\
& 10.0 & 15.5 & 103.3 & 2.9 \\
Urine & 0 & - & - & - \\
& 5.0 & 4.9 & 98.0 & 2.7 \\
& 10.0 & 10.3 & 103.0 & 1.8 \\
& 15.0 & 15.2 & 101.3 & 2.4 \\
\hline
\end{tabular}

\section{Conclusion}

In this study, $\mathrm{Ag} / \mathrm{ZnO}$ nanoplates were synthesized. An original sensing layer was fabricated by incorporating the assembled $\mathrm{Ag} / \mathrm{ZnO}$ nanoplates within a glassy carbon electrode to determination of zolpidem. When the peak currents were plotted against the analyte concentrations within the $0.1 \mu \mathrm{M}$ to $500.0 \mu \mathrm{M}$ range, the linear calibration curve was acquired. The zolpidem detection limit was $0.03 \mu \mathrm{M}$. The resulting electrode displayed favorable results when used to determine the zolpidem within the real samples.

\section{Acknowledgements}

The authors acknowledge the financial support provided for this project (Project No. 98000329 and ethics code IR.KMU.REC.1398.238) by theKerman University of Medical Sciences, Kerman, Iran.

\section{References}

[1] M. Mahajan, S. Sawant, Int. J. Chem. Tech. Res., 2012, 4, 403-408. 
[2] S.D. Radha, K.C.A. Rao, K.R. Krishna, Int. J. Chem. Tech. Res., 2016, $5,1315$.

[3] M.P. Mahajan, S.D. Sawant, Int. J. Pharm. Pharm. Sci., 2012, 4, 268-274.

[4] M. Jang, H. Chang, W. Yang, H. Choi, E. Kim, B.H. Yu, Y. Oh, H. Chung, J. Pharm. Biomed. Anal., 2013, 74, $213-$ 222.

[5] B. Lemmer, Physiol. Behav., 2007, 90, 285-293.

[6] L. Laviana, C. Mangas, F. FernándezMarí, M. Bayod, D. Blanco, J. Pharm. Biomed. Anal., 2004, 36, 925-928.

[7] C. Sasaki, T. Shinozuka, C. Murakami, W. Irie, K. Maeda, T. Watanabe, N. Nakamaru, M. Furukawa, S. Nakamura, K. Kurihara, Forensic Sci. Int., 2013, 227, 90-94.

[8] A. Mokhtari, M. Aaghamohammadhasan, Eurasian $J$. Anal. Chem., 2017, 12, 61-74.

[9] R. Chomwal, A. Kumar, A. Goyal, J. Pharm. Bioallied. Sci., 2010, 2, 365-368. [10] I. De-Clerck, P. Daenens, Analyst, 1997, 122, 1119-1124.

[11] F. Stanke, N. Jourdil, J. Bessard, G. Bessard, J. Chromatogr. B Biomed. Sci. Appl., 1996, 675, 43-51.

[12] A. Naeemy, E. Sedighi, A. Mohammadi, J. Electrochem. Sci. Technol., 2016, 7, 68-75.

[13] A.E. Radi, G. Bekhiet, T. Wahdan, Chem. Pharm. Bull., 2004, 52, 1063-1065.

[14] H. Mahmoudi-Moghaddam, H. Beitollahi, S. Tajik, I. Sheikhshoaie, P. Biparva, Environ. Monit. Assess., 2015, 187, 407-418.

[15] H. Karimi-Maleh, C.T. Fakude, N. Mabuba, G.M. Peleyeju, O.A. Arotiba, J. Colloid Interface Sci., 2019, 554, 603610.

[16] F. Tahernejad-Javazmi, M. ShabaniNooshabadi, H. Karimi-Maleh, Composites Part B, 2019, 172, 666-670.
[17] S. Esfandiari-Baghbamidi, $\mathrm{H}$. Beitollahi, S. Tajik, Anal Bioanal. Electrochem., 2014, 6, 634-645.

[18] A. Khodadadi, E. Faghih-Mirzaei, H. Karimi-Maleh, A. Abbaspourrad, S. Agarwal, V. K. Gupta, Sens. Actuators B, 2019, 284, 568-574.

[19] H. Karimi-Maleh, M. Sheikhshoaie, I. Sheikhshoaie, M. Ranjbar, J. Alizadeh, N. Wendy Maxakato, A. Abbaspourrad, New J. Chem., 2019, 43, 2362-2367.

[20] M. M. Foroughi, H. Beitollahi, S. Tajik, A. Akbari, R. Hosseinzadeh, Int. J. Electrochem. Sci., 2014, 9, 8407-8421.

[21] M. Miraki, H. Karimi-Maleh, M. A.Taher, S. Cheraghi, F. Karimi, S. Agarwal, V. K. Gupta, J. Mol. Liq., 2019, 278, 672-676.

[22] S. Ramki, P. Balasubramanian, S. M. Chen, T. W. Chen, T. W. Tseng, B. S. Lou, Int. J. Electrochem. Sci., 2018, 13, 1241-1249.

[23] S.K. Mittal, S. Rana, N. Kaur, C.E. Banks, Analyst, 2018, 143, 2851-2861.

[24] T.L. Lu, Y.C. Tsai, Sens. Actuators B-Chem., 2011, 153, 439-444.

[25] A. Özcan, S. İlkbaş, Sens. Actuators B-Chem., 2015, 215, 518-524.

[26] H. Beitollahi, H. Karimi-Maleh, H. Khabazzadeh, Anal. Chem., 2008, 80, 9848-9851.

[27] F. Ahmadi, E. Karamian, Iran J. Pharm. Res., 2014, 13, 417-429.

[28] H. Mahmoudi-Moghaddam, S. Tajik, H. Beitollahi, Food Chem., 2019, 286, 191-196.

[29] M.M. Shahid, P. Rameshkumar, N.M. Huang, Microchim. Acta, 2016, 183, 911-916.

[30] H. Beitollahi, S. Tajik, Environ. Monit. Assess., 2015, 187, 257-267.

[31] H. Heli, Iran. J. pharm. Res., 2015, 14, 453-463.

[32] A. Nowroozi, P. Mohammadzadeh Jahani, N. Asli, H. Hajiabadi, S. Dahmardeh, . Raissi, Int. J. Quantum Chem., 2012, 112, 489-497. 
[33] H. Soltani, H. Beitollahi, A.H. Hatefi-Mehrjardi, S. Tajik, M. Torkzadeh-Mahani, Anal. Bioanal. Electrochem., 2014, 6, 67-79.

[34] A. Nowroozi, H. Roohi, M. Poorsargol, P. Mohammadzadeh Jahani, H. Hajiabadi, Int. J. Quantum Chem., 2011,111, 3008-3016.

[35] A. Afkhami, A. Bahiraei, T. Madrakian, Mater. Sci. Eng. C., 2016, 59, 168-176.

[36] S. Esfandiari-Baghbamidi, $\mathrm{H}$. Beitollahi, S. Tajik, R. Hosseinzadeh, Int. J. Electrochem. Sci., 2016, 59, 10874-10883.

[37] P. Mohammadzadeh Jahani, A. Nowroozi, H. Hajiabadi, M. Hassani, Structural Chem., 2012, 23, 1941-1950. [38] F. Safari, M. Keyvanfard, H. Karimi-Maleh, $\quad$ K. Alizad, Iran. J. Pharm. Res., 2017, 16, 1019-1029.

[39] M.M. Motaghi, H. Beitollahi, S. Tajik, R. Hosseinzadeh, Int. J. Electrochem. Sci., 2016, 11, 7849-7860. [40] M.R. Ganjali, H. Beitollahi, R. Zaimbashi, S. Tajik, M. Rezapour, B. Larijani, Int. J. Electrochem. Sci., 2018, 13, 2519-2529.

[41] G. Karim-Nezhad, A. Sarkary, Z. Khorablou, P.S. Dorraji, Iran. J. Pharm. Res., 2018, 17, 52-62.

How to cite this manuscript: Mohammad Reza Aflatoonian, Behnaz Aflatoonian, Reza Alizadeh, Reza Abbasi Rayeni. Voltammetric determination of zolpidem by using glassy carbon electrode modified with $\mathrm{Ag} / \mathrm{ZnO}$ nanoplates. Eurasian Chemical Communications, 2020, 2(1), 35-43.
[42] M.R. Ganjali, H. Salimi, S. Tajik, H. Beitollahi, M. Rezapour, B. Larijani, Int. J. Electrochem. Sci., 2017, 12, 52432553.

[43] M. Eldefrawy, E.G.A. Gomaa, S. Salem, F. Abdel Razik, Prog. Chem. Biochem. Res. 2018, 1, 11-18.

[44] S. Mohammadi, A. Taheri, Z. Rezayati-Zad,. Prog. Chem. Biochem. Res. 2018, 1, 1-10.

[45] G. Vinodhkumar, R. Ramya, M. vimalan, I. Potheher, A. Cyrac Peter, Prog. Chem. Biochem. Res. 2018, 1, 4049.

[46] A. Foda, H. Mosallam, A. ElKhateeb, M. Fakih, Prog. Chem. Biochem. Res., 2019, 2, 120-133.

[47] S.M. Botsa, R. Dharmasoth, K. Basavaiah, Current Nanosci., 2019, 15, 209-213.

[48] H. Thacker, V. Ram, P.N. Dave,. Prog. Chem. Biochem. Res. 2019, 2, 8491.

[49] H. Beitollahi, A. Gholami, M.R. Ganjali, Mater. Sci. Eng. C, 2015, 57, 107-112.

[50] A.J. Bard, L. R. Faulkner, second ed., Wiley, New York, NY, 2001. 